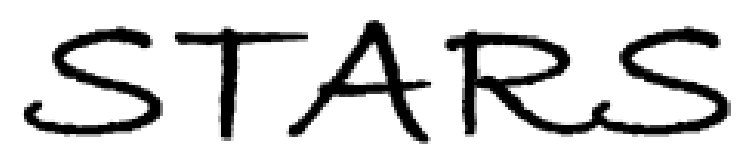

University of Central Florida

STARS

$1-1-1997$

\title{
The renormalization group and dynamical triangulations
}

Ray L. Renken

University of Central Florida

Find similar works at: https://stars.library.ucf.edu/facultybib1990

University of Central Florida Libraries http://library.ucf.edu

This Article; Proceedings Paper is brought to you for free and open access by the Faculty Bibliography at STARS. It has been accepted for inclusion in Faculty Bibliography 1990s by an authorized administrator of STARS. For more information, please contact STARS@ucf.edu.

\section{Recommended Citation}

Renken, Ray L., "The renormalization group and dynamical triangulations" (1997). Faculty Bibliography 1990s. 2071.

https://stars.library.ucf.edu/facultybib1990/2071

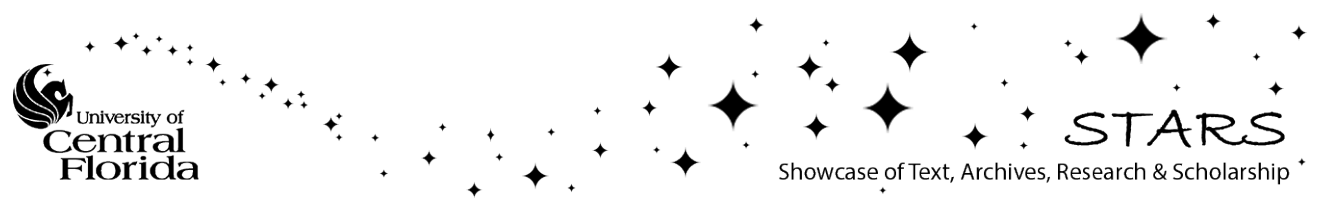




\title{
The Renormalization Group and Dynamical Triangulations
}

\author{
R. L. Renken ${ }^{\text {a }}$
}

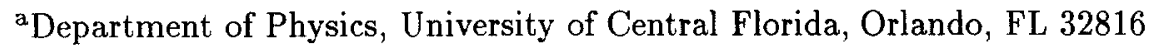

A block spin renormalization group approach is introduced which can be applied to dynamical triangulations in any dimension.

\section{INTRODUCTION}

Given a general action for a theory of dynamical triangulations, two limits must be taken to obtain a physical theory. The cosmological constant must be adjusted to give the infinite volume limit (or some finite target volume in practical calculations). The remaining parameters in the action must be used to find a second order phase transition so that a continuum limit of the lattice theory can be taken. The renormalization group approach is a natural technique with which to search for and study the required critical phenomena [1-4].

The renormalization group approach can be viewed as a black box that takes some initial theory $S$ with correlation length $\xi$ and produces an effective theory $S^{\prime}$ with correlation length $\xi^{\prime}=$ $\xi / b . \quad b$ is fixed and is a property of the chosen renormalization group transformation. By taking the output of the block box and feeding it back into the input, it is possible to produce a sequence of theories: $S^{(0)}, S^{(1)}, S^{(2)}, \cdots$ with a corresponding sequence of correlation lengths $\xi^{(0)}$, $\xi^{(1)}, \xi^{(2)}, \ldots$ If the original theory has a finite correlation length, the $\xi^{(n)} \rightarrow 0$ as the renormalization group transformation is iterated. Such a theory is referred to as a trivial theory. If the original theory has an infinite correlation length, as occurs at a second order phase transition, then the correlation length stays infinite after each renormalization group transformation and what happens is that the effective theory approaches a fixed point, $S^{(n)} \rightarrow S^{*}$.

In an ordinary statistical mechanical model, such as the Ising model, the degrees of freedom can be organized into fixed blocks and then aver- aged according to some rule to produce a block degree of freedom. If the blocks are, for instance, of volume $b^{D}$, then the scale factor is $b$ as above. Dynamical triangulations are different in that there is apparently no way to draw fixed boxes around the degrees of freedom, which are determined by the connectivity of the lattice. A related difficulty is that the Hausdorff dimension is not known a priori so that rescaling a volume by some factor does not determine what the length is rescaled by. A related difficulty is that the number of configurations cannot be enumerated in the trivial way they can for ordinary statistical mechanical models. In fact, for three and four dimensions, it is not even known with certainty that this number grows only exponentially with the volume.

A dynamical triangulation is interpreted as a lattice representation of a spacetime with a metric. Nodes connected by a link are considered closer than those that are not connected by a link. One way to define a renormalization group transformation for dynamical triangulations is to insist that the block triangulation preserve this physical notion. Such a transformation succeeds at getting critical couplings in two dimensions, but exponent calculations fail to converge and the method is difficult to generalize to arbitrary $D[5,6]$.

Another renormalization group transformation in two dimensions is based on the idea of removing a node and its associated triangles from the lattice manifold and filling the hole back in with triangles but without any new nodes [7]. This is achieved by making flips of links connected to the node in question until its coordination number is three. It and its three associated triangles are then removed and replaced with a single trian- 


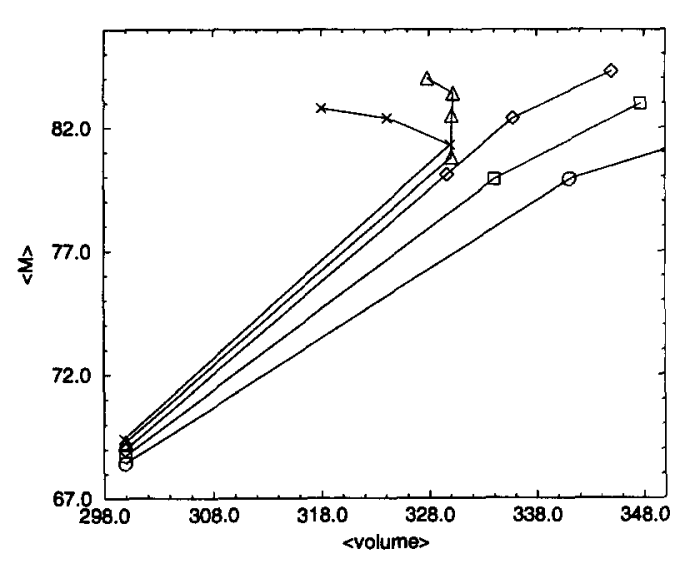

Figure 1. Renormalization group flows in the four-dimensional dynamical triangulation model near the transition.

gle. This method correctly produces both critical couplings and critical exponents.

It is possible to make this approach work in arbitrary dimensions [8]. In general $D$, the possible update moves can be labeled by $i$ with $i=0,1, \cdots, D$. An update move labeled by $i$ swaps an $i$-dimensional subsimplex of a randomly chosen simplex with a $(D-i)$-dimensional subsimplex. This eliminates $D-2 i$ simplexes. In two dimensions, a link flip is an $i=1$ move while node deletion is an $i=0$ move. In a two-dimensional ensemble with fixed volume these are the only choices. In order to eliminate a node in general dimensions, some algorithm for choosing $i$ is necessary since there are more possible values. It is best to use moves with the lowest value of $i$ possible, since these remove the most simplexes. $i=0$ is only possible on the last move (when the node has coordination number $D+1$ ) and $i=1$ is not always possible due to geometrical constraints and the existence of connections on the surface around the node. In fact, it is sometimes necessary to make an $i=D$ move, which is node insertion.

While deletion of a single node is viewed as a renormalization group transformation resulting in a block lattice, it is generally preferable to delete a number of nodes. In order to eliminate finite size effects, it is desirable to block all lattices down to the same target number of nodes. As the volume of the initial lattice is varied, the resulting blocked theories embody the physics at a range of length scales. Two operators are used here to track the renormalization group flows: the volume and a term $M$ defined by

$$
M=\sum_{i \in N_{0}} \ln \left(\frac{O_{i}}{D+1}\right)
$$

where $O_{i}$ is the number of simplexes containing the node $i$. If $M$ is added to the action

$$
S_{M}=\mu M
$$

it corresponds to the addition of a measure term [9].

The model of dynamical triangulations considered first has the action

$$
S=\alpha N_{0}-\beta N_{D}
$$

where $D$ is either three or four [10]. $N_{0}$ is the number of nodes with $\alpha$ corresponding to Newton's constant while $N_{D}$ is the volume so that $\beta$ is the cosmological constant. Figure 1 shows a plot of $\langle M\rangle$ versus the volume as a function of the degree of blocking for various values of $\alpha$ in four dimensions. The renormalization group flows for the smaller values of $\alpha$ flow toward the right, toward decreasing node density, which is the expected behavior in the crumpled phase. For larger $\alpha$ flows are to the left, a different behavior, indicative of a flow toward a different trivial fixed point, presumably associated with the smooth phase. The presence of an intermediate type of flow suggests that a non-trivial fixed point may be nearby.

In three dimensions, shown in Fig. 2, there are similar flows to the left and to the right, but no intermediate flow. This is consistent with the strongly first order character of the transition.

The phase diagram of both the threedimensional and four-dimensional theories can be expanded by adding the previously defined measure term to the action. Recent work indicates 


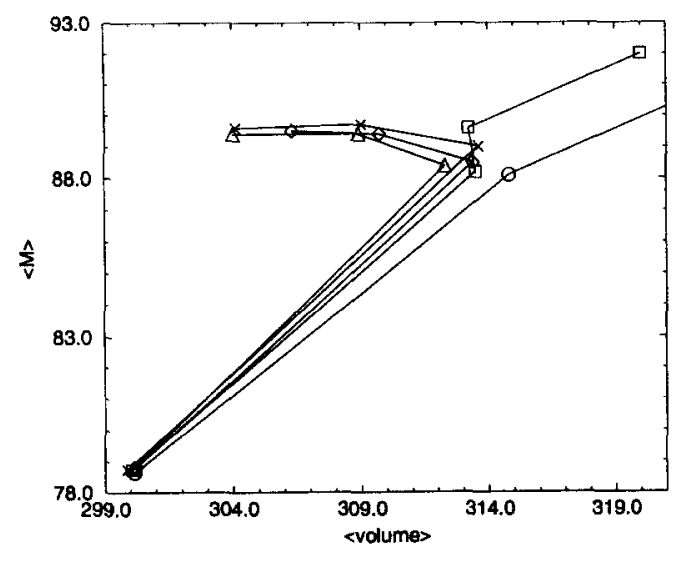

Figure 2. Renormalization group flows in the three-dimensional dynamical triangulation model near its transition.

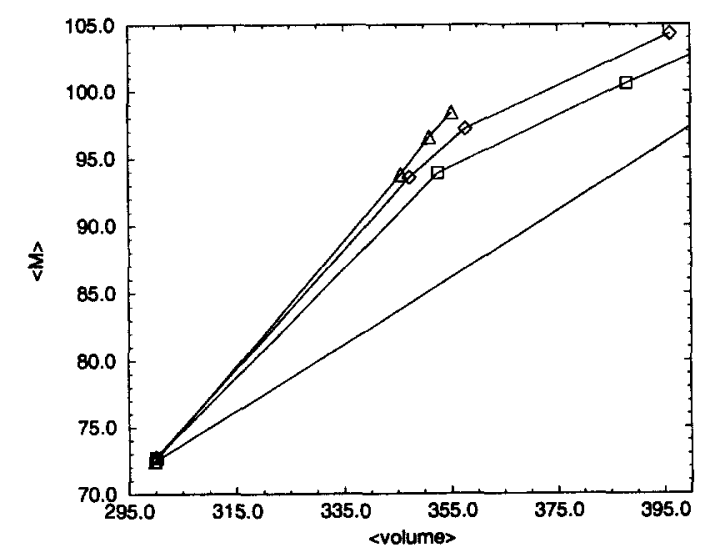

Figure 3. Renormalization group flows in the three-dimensional dynamical triangulation model with a measure term included. that the $\mu=0$ transitions are a part of a first order line in the $(\alpha, \mu)$ plane [11]. If this line terminates in a critical endpoint, that would provide the second order phase transition required for taking a continuum limit. This talk considers whether there is still a transition at $\mu=-1$ in three dimensions. Flows for various values of $\alpha$ are given in Fig. 3. There is no evidence of a transition. More recent work at very large values of $\alpha$ has discovered flows like that of the smooth phase [12], but that is not necessarily inconsistent with behavior past the end of a first order line. More work will be necessary to establish that the first order line actually ends.

\section{REFERENCES}

1. K. G. Wilson, Rev. Mod. Phys. 47, 773 (1975).

2. K. G. Wilson, in Recent Developments in Gauge Theories, ed. G 't Hooft (Plenum Press, N. Y. 1980).

3. M. E. Fisher, Scaling, Universality and Renormalization Group Theory, in Critical Phenomena Lecture Notes in Physics, Vol. 186, ed F. J. W. Hahne (Springer-Verlag, Berlin, 1983).

4. G. S. Pawley, R. H. Swendsen, D. J. Wallace, and K. G. Wilson, Phys. Rev. B29, 4030 (1984).

5. R. L. Renken, Phys. Rev. D50, 5130 (1994).

6. R. L. Renken, S. M. Catterall, and J. B. Kogut, Phys. Lett. B345, 422 (1995).

7. G. Thorleifsson and S. M. Catterall, SU-4240619.

8. S. M. Catterall, Computer Physics Comm. 87, 409, 1995.

9. B. Brugmann and E. Marinari, Phys. Rev. Lett. 70, 1908, 1993.

10. S. Catterall, "Lattice Quantum Gravity: Review and Recent Developments," heplat/9510008 and D. Johnston, "Gravity and Random Surfaces," hep-lat/9607021.

11. S. Catterall, J. Kogut, and R. Renken, manuscript in preparation.

12. R. L. Renken, "A Renormalization Group for Dynamical Triangulations in Arbitrary Dimensions," hep-lat/9607074. 